\title{
A Case of Deep Vein Thrombosis and Intracranial Sinus Thrombosis Possible rare complications of childhood abdominal tuberculosis
}

Geeta Gathwala, *Poonam Dalal, Virender Gehlawat, Jasbir Singh, Vandana Arya

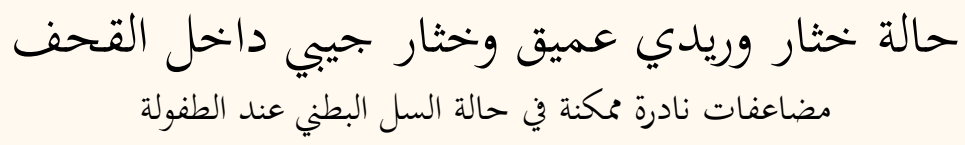

جيتا جاتواثلا، بونام دلال، فيريندر جيلاوات، جاسبر سينج، فاندانا أريا

ABSTRACT: Severe pulmonary tuberculosis (TB) complicated by deep vein thrombosis (DVT) in adults has been reported previously in the medical literature; however, childhood extrapulmonary TB complicated by DVT is rare. We report a 13-year-old girl who presented to the Department of Pediatrics at the Postgraduate Institute of Medical Sciences in Rohtak, India, in 2012 with abdominal TB complicated by DVT and intracranial sinus thrombosis. She was treated with a course of four antitubercular drugs and short-term anticoagulation therapy with a positive outcome over the next six months. To the best of the authors' knowledge, no previous reports have yet suggested a possible association between childhood TB and intracranial sinus thrombosis.

Keywords: Deep Vein Thrombosis; Intracranial Sinus Thrombosis; Gastrointestinal Tuberculosis; Case Report; India.

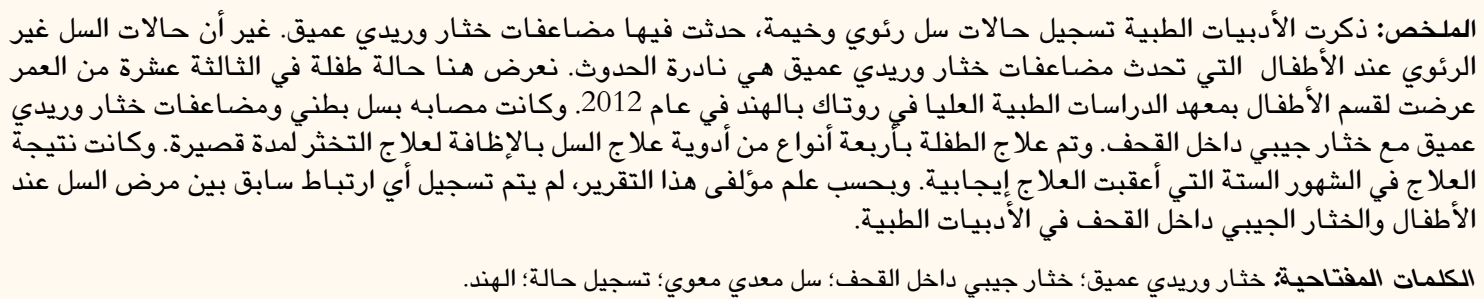

A HYPERCOAGULABLE STATE HAS BEEN DESCribed in patients with tuberculosis (TB), with a reported incidence of deep vein thrombosis (DVT) in 3-4\% of adults with pulmonary TB. ${ }^{1,2}$ However, cases of extrapulmonary TB complicated by thrombotic events are rare and only two cases of childhood abdominal TB complicated by DVT have been previously described in the literature. ${ }^{3,4}$ Moreover, TB complicated by cerebral venous thrombosis is very rarely reported. ${ }^{5}$ To the best of the authors' knowledge, this is the first report of a case of childhood TB with intracranial sinus thrombosis.

\section{Case Report}

A 13-year-old female presented to the Department of Pediatrics at the Postgraduate Institute of Medical Sciences in Rohtak, India, in 2012 with a five-month history of intermittent fever and pain in the abdomen. The fever was of a low-to-moderate grade and became more pronounced in the evening, while the abdominal pain was diffuse and moderate in intensity. There was a history of reduced appetite and a documented weight loss of $14 \mathrm{~kg}$ over the previous five months. The patient had no history of contact with known cases of TB. She had been immunised with the bacillus CalmetteGuérin vaccine at birth.

At presentation, the patient was pale and emaciated. Her weight was $24 \mathrm{~kg}$ and her height was 124 $\mathrm{cm}$ (both values below the $3^{\text {rd }}$ centile for her age). The abdomen was distended with tenderness in the right iliac fossa and a palpable lump. The liver was non-tender with a span of $10 \mathrm{~cm}$. The results of blood investigations were as follows: haemoglobin count of $6.7 \mathrm{~g} / \mathrm{dL}$; leukocyte count of $7,000 / \mathrm{mm}^{3}$; differential of $60 \%$ polymorphs and $36 \%$ lymphocytes; platelet count of $250,000 / \mathrm{mm}^{3}$; a dimorphic blood picture with predominantly microcytic anaemia; and an erythrocyte sedimentation rate of $50 \mathrm{~mm} /$ hour. Her Mantoux test result with 5 tuberculin units was $18 \mathrm{~mm} / 72$ hours. Liver and renal function tests were normal and a human immunodeficiency virus 


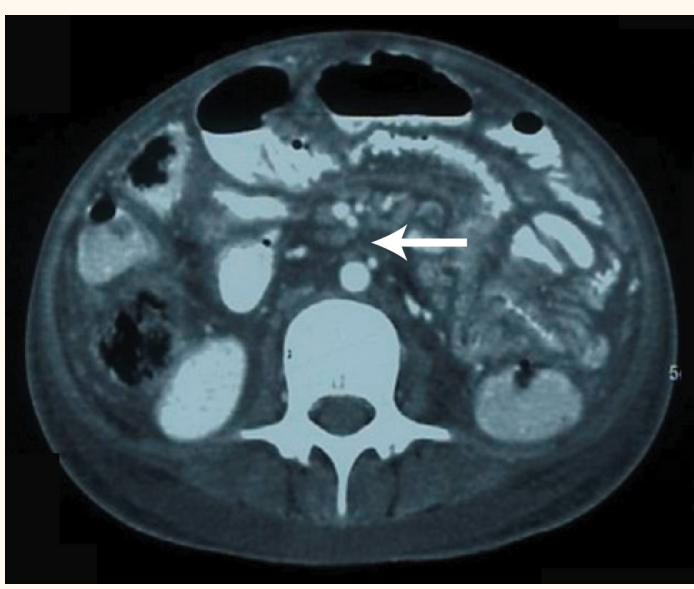

Figure 1: Contrast-enhanced computed tomography scan of the abdomen of a 13-year-old girl with intermittent fever and abdomen pain showing enlarged lymph nodes (arrow) in the pre-aortic region and stranding of the mesentery.

serology test was non-reactive. Her serum protein levels were $5.1 \mathrm{~g} / \mathrm{dL}$. While a chest X-ray was normal, an abdominal X-ray revealed multiple air-fluid levels. Ultrasonography of the abdomen revealed free fluid, matting of the bowel loops, pelvic septations and multiple enlarged mesenteric nodes. A computed tomography scan of the abdomen revealed enlarged mesenteric lymph nodes with stranding of the mesentery [Figure 1], indicating a tubercular aetiology. There was no evidence of compression of the inferior vena cava by the lymph nodes. A gastric lavage was positive for acid-fast bacilli. Consequently, a diagnosis of probable abdominal TB was made and the patient was prescribed four-drug antitubercular therapy three days after admission, comprised of rifampicin, isoniazid, pyrazinamide and ethambutol.

Later that day, the patient developed pain and swelling in the left leg and thigh. There was evidence

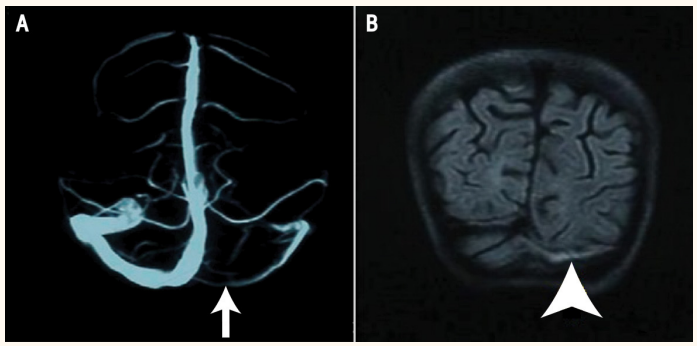

Figure 2: A: Magnetic resonance (MR) venogram of the brain of a 13-year-old girl with probable abdominal tuberculosis and sudden-onset altered sensorium, aphasia and right-sided haemiparesis. Note the thinning of the left transverse sinus (arrow), suggestive of intracranial sinus thrombosis. B: T2-weighted fluidattenuated inversion recovery MR image showing hyperintensity in the region of the thrombosed left transverse sinus (arrowhead). of pitting oedema and swelling, along with calf tenderness. On ultrasonography, the common femoral, superficial, deep femoral and popliteal veins were non-compressible with echogenic thrombi. No flow was observed on a colour Doppler ultrasound, confirming the diagnosis of DVT. A detailed coagulation profile revealed protein $\mathrm{C}$ levels of $68 \%$ (reference value $[R V]$ : $60-120 \%)$, protein $\mathrm{S}$ levels of $77 \%$ (RV: 60-120\%), D-dimer levels of $137 \mathrm{ng} / \mathrm{mL}$ (RV: $<500 \mathrm{ng} / \mathrm{mL}$ ), prothrombin time of 15 seconds (RV: 13 seconds), activated partial thromboplastin time of 34 seconds (RV: 28 seconds) and an international normalised ratio (INR) of 1.2. Levels of fibrin degradation products were $3.6 \mu \mathrm{g} / \mathrm{mL}$ (RV: $0-1.34 \mu \mathrm{g} / \mathrm{mL}$ ) while antithrombin III levels were 62\% (RV: 80-130\%). Test results for factor V Leiden and antinuclear antibodies were normal. The patient was administered low-molecular-weight heparin and warfarin. The warfarin was initially prescribed at a dose of $0.1 \mathrm{mg} / \mathrm{kg} /$ day with the dose adjusted every third day to achieve an INR of 2-3. The INR was frequently monitored to avoid the possible enzyme induction effects of rifampicin. ${ }^{1}$

On the tenth day of admission, the patient developed sudden-onset altered sensorium, aphasia and right-sided haemiparesis. A lumbar puncture was performed to rule out tubercular meningitis; how-ever, examination of the cerebrospinal fluid revealed no abnormalities. A magnetic resonance imaging (MRI) scan of the brain revealed no abnormal findings in the brain parenchyma. There was no evidence of ventricular enlargement, basal exudates or infarcts. There was a hyperintense signal in the left transverse sinus on a T2-weighted fluid attenuated inversion recovery MRI scan. In addition, there was a narrowing of the corresponding sinus on a magnetic resonance venogram [Figure 2], suggestive of intracranial sinus thrombosis. After excluding systemic causes or seizures as possible causes of the altered sensorium and focal deficits, the possibility of cerebral vein thrombosis was considered. The altered sensorium improved over the following two days and the haemiparesis improved over the next 5-7 days, without any residual neurological deficits.

The patient made an uneventful recovery while undergoing the antitubercular and anticoagulation therapy. She became afebrile within two weeks, while the limb oedema and pain improved within four weeks. Her fibrinogen degradation product and antithrombin III levels were normal at four weeks. A repeat ultrasound of the left leg revealed a residual thrombus; however, evidence of partial re-canalisation and flow on a colour Doppler MRI suggested that it was in the process of resolving. She continued to take 
low-molecular-weight heparin for 10 days, warfarin for three months and antitubercular drugs for six months. Regular follow-up appointments over the next six months indicated that she was in good health and had gained $11 \mathrm{~kg}$. Unfortunately, the patient was subsequently lost to follow-up.

\section{Discussion}

Severe TB is known to induce an acute phase response which leads to the activation of mononuclear cells; the interaction of mycobacterial products with activated mononuclear cells subsequently induces increased synthesis of tumour necrosis factor- $\alpha$ and interleukin-6. ${ }^{6,7}$ These proinflammatory cytokines activate the vascular intima, rendering the endothelial surface thrombogenic. The cytokines also induce a hepatic acute phase response leading to deranged levels of coagulation proteins and a subsequent hypercoagulable state. ${ }^{6,7}$ There are very few reports of thrombotic complications among children with TB, possibly due to under-reporting. ${ }^{3,4}$ This type of rare but serious complication can further increase the morbidity and mortality associated with TB in young children; ${ }^{8}$ as such, clinicians should keep a high index of suspicion of DVT. To the best of the authors' knowledge, this is the first reported case of childhood abdominal TB with DVT and intracranial sinus thrombosis.

Robson et al. reported 35 cases of pulmonary TB and DVT in adults; of these, venous thrombosis occurred seven days after diagnosis for 33 patients, while two had DVT upon presentation. ${ }^{6}$ The patient in the present case developed symptoms of DVT on the third day of admission, before the commencement of antitubercular therapy. Elevated plasma fibrinogen, impaired fibrinolysis, decreased levels of antithrombin III and reactive thrombocytosis can lead to the development of DVT among patients with pulmonary TB. ${ }^{6}$ A hypercoagulable state in patients with TB has been reported to improve within four weeks with the use antitubercular drugs. ${ }^{7}$ In the current case, the patient had elevated levels of fibrin degradation products and decreased levels of antithrombin III which resolved within a month of antitubercular therapy.

TB can predispose to DVT due to a hypercoagulable state. ${ }^{6}$ Retroperitoneal tubercular lymphadenitis leading to compression of the inferior vena cava has also been reported to cause DVT in the absence of any haemostatic abnormalities. ${ }^{9}$ Immobility can further exacerbate the effects of a hypercoagulable state. Although the patient in the present case was diagnosed with probable abdominal TB with mesenteric lymphadenitis, there was no evidence of inferior vena cava compression. Thus, the DVT and intracranial sinus thrombosis were possibly due to the patient's hypercoagulable state.

Isoniazid and rifampicin can exacerbate the thrombogenic potential of TB by killing mycobacteria and enhancing the release of interleukin- 6 from the peripheral blood mononuclear cells. ${ }^{1}$ Rifampicin is a potent inducer of cytochrome $\mathrm{P} 450$ proteins and thus enhances metabolism of oral anticoagulants; ${ }^{1}$ as a result, adjustments to the dosage of warfarin may be necessary to maintain its therapeutic efficacy. Doses and durations of anticoagulant treatment for patients with TB have not yet been standardised. The recommended duration of anticoagulants for children with secondary and idiopathic venous thrombosis is three and six months, respectively. ${ }^{10}$ It has been suggested that the hypercoagulable state and haemostatic changes stabilise within four weeks of antitubercular therapy. ${ }^{7}$ In the current patient, the DVT resolved within a month of antitubercular treatment, which provides further evidence of a correlation between TB and venous thrombosis.

\section{Conclusion}

While DVT is a rare complication associated with pulmonary and extrapulmonary TB in adult patients, reports of this complication occurring with childhood TB are scarce. There is a need to keep a high index of suspicion of this rare but serious complication as it can further increase the morbidity and mortality associated with TB in young children. In addition, the current case highlights for the first time a possible association between childhood TB and intracranial sinus thrombosis.

\section{References}

1. White NW. Venous thrombosis and rifampicin. Lancet 1989; 2:434-5. doi: 10.1016/S0140-6736(89)90603-X.

2. Ambrosetti M, Ferrarese M, Codecasa LR, Besozzi G, Sarassi A, Viggiani P, et al. Incidence of venous thromboembolism in tuberculosis patients. Respiration 2006; 73:396. doi: 10.1159/ 000091188 .

3. Gupta R, Brueton M, Fell J, Lyall H. An Afghan child with deep vein thrombosis. J R Soc Med 2003; 96:289-91. doi: 10.1258/ jrsm.96.6.289.

4. Havaldar PV, Mogale KD, Koppad AM, Haravi R. Spontaneous deep vein thrombosis. Indian Pediatr 1998; 35:657-63.

5. Fiorot Júnior JA, Felício AC, Fukujima MM, Rodrigues CA, Morelli VM, Lourenço DM, et al. Tuberculosis: An uncommon cause of cerebral venous thrombosis? Arq Neuropsiquiatr 2005; 63:852-4. doi: 10.1590/S0004-282X2005000500025. 
6. Robson SC, White NW, Aronson I, Woollgar R, Goodman H, Jacobs P. Acute-phase response and the hypercoagulable state in pulmonary tuberculosis. Br J Haematol 1996; 93:943-9. doi: 10.1046/j.1365-2141.1996.d01-1722.x.

7. Turken O, Kunter E, Solmazgul E, Cerrahoglu K, Bozkanat E, Ozturk A, et al. Hemostatic changes in active pulmonary tuberculosis. Int J Tuberc Lung Dis 2002; 6:927-32.

8. Dentan C, Epaulard O, Seynaeve D, Genty C, Bosson JL. Active tuberculosis and venous thromboembolism: Association according to International Classification of Diseases, ninth revision hospital discharge diagnosis codes. Clin Infect Dis 2014; 58:495-501. doi: $10.1093 / \mathrm{c} \mathrm{id/cit780.}$
9. Gogna A, Pradhan GR, Sinha RS, Gupta B. Tuberculosis presenting as deep vein thrombosis. Postgrad Med J 1999; 75:104-6. doi: $10.1136 /$ pgmj.75.880.104.

10. Chalmers E, Ganesen V, Liesner R, Maroo S, Nokes T, Saunders D, et al. Guideline on the investigation, management and prevention of venous thrombosis in children. Br J Haematol 2011; 154:196-207. doi: 10.1111/j.1365-2141.2010.08543.x. 